\title{
Ballistic supercurrent discretization and micrometer-long Josephson coupling in germanium
}

\author{
N. W. Hendrickx,,${ }^{1, *}$ M. L. V. Tagliaferri, ${ }^{1}$ M. Kouwenhoven, ${ }^{1}$ R. Li,${ }^{1}$ D. P. Franke,${ }^{1}$ A. Sammak, ${ }^{2}$ \\ A. Brinkman, ${ }^{3}$ G. Scappucci, ${ }^{1}$ and M. Veldhorst ${ }^{1, \dagger}$ \\ ${ }^{1}$ QuTech and Kavli Institute of Nanoscience, Delft University of Technology, P.O. Box 5046, 2600 GA Delft, Netherlands \\ ${ }^{2}$ QuTech and the Netherlands Organisation for Applied Scientific Research (TNO), Stieltjesweg 1, 2628 CK Delft, Netherlands \\ ${ }^{3}$ Faculty of Science and Technology and MESA+ Institute for Nanotechnology, University of Twente, 7500 AE Enschede, Netherlands
}

(Received 17 December 2018; revised manuscript received 7 February 2019; published 27 February 2019)

\begin{abstract}
We fabricate Josephson field-effect transistors in germanium quantum wells contacted by superconducting aluminum and demonstrate supercurrents carried by holes that extend over junction lengths of several micrometers. In superconducting quantum point contacts we observe discretization of supercurrent, as well as Fabry-Pérot resonances, demonstrating ballistic transport. The magnetic field dependence of the supercurrent follows a clear Fraunhofer-like pattern, and Shapiro steps appear upon microwave irradiation. Multiple Andreev reflections give rise to conductance enhancement and evidence a transparent interface, confirmed by analyzing the excess current. These demonstrations of ballistic superconducting transport are promising for hybrid quantum technology in germanium.
\end{abstract}

DOI: 10.1103/PhysRevB.99.075435

\section{INTRODUCTION}

Quantum information processing in the solid state is being pursued using superconducting and semiconducting platforms $[1,2]$. In both platforms, rudimentary quantum algorithms have already been demonstrated $[3,4]$. While decoherence is a central topic, advanced superconducting systems are now capable of entangling ten qubits [5]. Spin qubits based on silicon and germanium, on the other hand, can be isotopically enriched to remove magnetic decoherence [6,7], resulting in extremely long coherence times $[8,9]$. Crucially, these qubits can be defined using conventional semiconductor technology. A hybrid approach may build upon the strengths of each platform, motivating extensive research. Superconducting qubits with semiconductor elements have led to electric gate-tunable superconducting qubits $[10,11]$, or gatemons, while spin qubits interfaced with superconducting resonators have reached the regime of strong spin-photon coupling [12-14], an important step toward long-range entanglement.

Furthermore, hybrid superconductor-semiconductor systems can host exotic excitations. In particular, a topological phase transition may occur in superconductor-semiconductor systems in the presence of spin-orbit coupling and magnetism $[15,16]$. At the topological transition, excitations emerge that represent Majorana fermion states that can exhibit nonAbelian exchange statistics. Next to their fundamental interest, these states are argued to be excellent building blocks for quantum computation as they bear topological protection against decoherence. Quantum information transfer between spin and topological qubits could make topological systems

\footnotetext{
*n.w.hendrickx@tudelft.nl

${ }^{\dagger}$ m.veldhorst@tudelft.nl
}

universal [17] and offer spin qubits topologically protected long-range links $[18,19]$.

Holes in germanium are an excellent material platform for the construction of these hybrid systems. Germanium can be isotopically purified, thereby removing decoherence by nuclear spins [6], and it can host strong spin-orbit coupling [20]. In addition, mobilities reaching $1500000 \mathrm{~cm}^{2} / \mathrm{V}$ s have been reported [21], and high-quality gate-defined quantum dots have been realized [22] in strained $\mathrm{SiGe} / \mathrm{Ge} / \mathrm{SiGe}$ heterostructures. Electrically driven spin qubits have been constructed [23], single spins can be read out in single-shot mode [24], and strained germanium quantum wells [25] have several additional favorable properties for spin qubit operation [26], including a small effective mass and large energy splitting to excited states. Gate-tunable superconductivity has been studied for zero- and one-dimensional hole systems in selfassembled Ge quantum dots [27] and in $\mathrm{Ge} / \mathrm{Si}$ nanowires [28], respectively. More recently, induced superconductivity was also observed in a two-dimensional (2D) Ge hole system [22], which greatly enhances the flexibility of the fabrication of nanostructures and allows quantum dots to be defined solely by lithography. Now it has to be determined whether ballistic phase-coherent superconductivity can be induced in the germanium platform over the length scales required for long-scale coupling in hybrid systems.

Here, we demonstrate gate-tunable Josephson supercurrents in a $2 \mathrm{D}$ germanium quantum well system with junction lengths $L$ up to $6 \mu \mathrm{m}$ and find a characteristic decay length $\xi^{*}=1.0 \mu \mathrm{m}$. In quantum point contacts we observe discretization of the supercurrent and conductance, demonstrating ballistic transport. From the excess current and multiple Andreev reflections we deduce an interface transparency $T$ between the leads and germanium of 0.6-0.7. Furthermore, we demonstrate the dc and ac Josephson effect in planar germanium via Fraunhofer-like patterns that arise in magnetic fields and Shapiro steps resulting from microwave irradiation. 

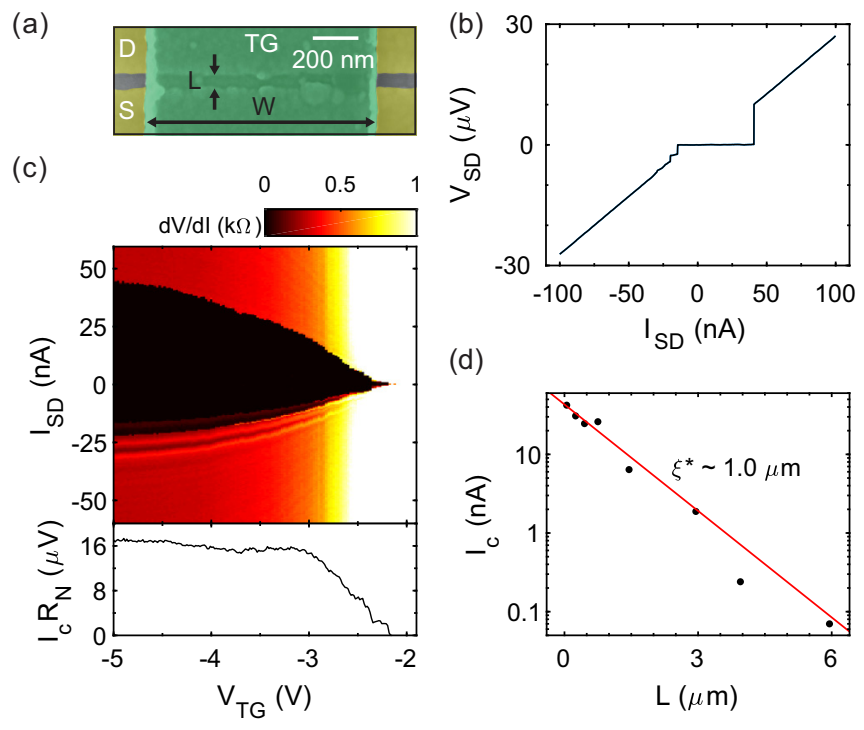

(d)

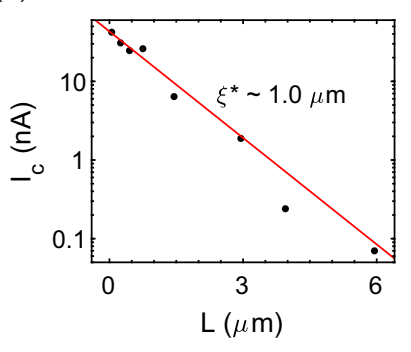

FIG. 1. (a) False-color SEM image of a planar Josephson junction device with width $W=1 \mu \mathrm{m}$ and length $L=50 \mathrm{~nm}$. The top gate (TG, indicated in green) is used to induce a $2 \mathrm{DHG}$ in the strained germanium quantum well contacted by a superconducting Al source (S) and drain (D) lead, depicted in yellow. (b) $I V$ curve of a junction with $L=50 \mathrm{~nm}$ at $V_{\mathrm{TG}}=-4 \mathrm{~V}$, showing a clear supercurrent with $I_{\mathrm{S}}=43 \mathrm{nA}$. (c) Color plot of the differential resistance of the junction $d V / d I$ as a function of the bias current $I_{\mathrm{SD}}$ and top-gate voltage $V_{\mathrm{TG}}$ (top panel) and derived $I_{\mathrm{S}} R_{\mathrm{N}}$ product as a function of $V_{\mathrm{TG}}$ (bottom panel). (d) Length dependence of the supercurrent. A purely exponential decay is observed over the entire junction length range, with a decay length of $1.0 \mu \mathrm{m}$.

\section{JOSEPHSON JUNCTIONS IN A GERMANIUM QUANTUM WELL}

\section{A. Micrometer-long supercurrents}

Figure 1(a) shows a scanning electron microscope (SEM) image of a germanium Josephson field-effect transistor (JoFET). The heterostructure is grown by reduced-pressure chemical vapor deposition and consists of a $\mathrm{Si}_{0.2} \mathrm{Ge}_{0.8}$ virtual substrate, a 16-nm-thick strained Ge quantum well, a 22-nmthick $\mathrm{Si}_{0.2} \mathrm{Ge}_{0.8}$ barrier, and, finally, a 1-nm-thick Si cap [25]. Superconducting leads are defined by thermal evaporation of aluminum after electron beam lithography and local etching of the Si capping layer. The accumulation top gate (TG) defining the junction is fabricated by depositing a titanium/palladium layer on top of an aluminum oxide dielectric layer grown by atomic layer deposition (ALD) at $300^{\circ} \mathrm{C}$. The JoFETs are fabricated with junction lengths $L$ between $50 \mathrm{~nm}$ and $6 \mu \mathrm{m}$. The Al contacts are $1 \mu \mathrm{m}$ wider than the width of the TG to ensure that superconductivity is present along the entire width of the junction.

A two-dimensional hole gas (2DHG) is formed by applying a negative gate voltage to the top gate, and a clear supercurrent becomes apparent in the $I V$ curve. Figure 1(b) shows a typical trace at a fridge base temperature of $T_{\text {bath }}=10 \mathrm{mK}$, where we find a switching current $I_{\mathrm{S}}=43 \mathrm{nA}$. The steps visible for negative bias are likely caused by self-induced Shapiro steps, as we discuss below. The switching current can be tuned by changing the hole density using the top gate, as shown in the top panel of Fig. 1(c), plotting the maximum out of ten switching events, in order to reduce the statistical spread in $I_{\mathrm{S}}$ and approximate $I_{\mathrm{C}}$. By measuring the normal-state resistance $R_{\mathrm{N}}$ at high bias, we determine the characteristic voltage $I_{\mathrm{S}} R_{\mathrm{N}}$ as a function of $V_{\mathrm{TG}}$, as shown in the bottom panel of Fig. 1(c). The observed value increases with more negative $V_{\mathrm{TG}}$ and saturates around $I_{\mathrm{S}} R_{\mathrm{N}} \approx 17 \mu \mathrm{V}$, comparable to values recently reported for Ge 2DHG devices [29]. Figure 1(d) shows the length dependence of $I_{\mathrm{S}}$ for $V_{\mathrm{TG}}=-4 \mathrm{~V}$, showing supercurrents with a purely exponential decay length $\xi^{*}=1.0 \mu \mathrm{m}$, extending over remarkably long length scales of several micrometers. For the junction with length $L=6.0 \mu \mathrm{m}$, we measure $I_{\mathrm{S}}=$ $70 \mathrm{pA}$, indicating the high quality of the quantum well. In comparison, supercurrents in semiconductors were reported over lengths up to $1.5 \mu \mathrm{m}$ in graphene [30], $1.6 \mu \mathrm{m}$ in GaAs [31], $2 \mu \mathrm{m}$ in InAs/GaSb [32], and $3.5 \mu \mathrm{m}$ in $\mathrm{Bi}_{2} \mathrm{Te}_{3}$ [33]. From Hall bar magnetotransport data, we derive a carrier mobility $\mu=500000 \mathrm{~cm}^{2} / \mathrm{V} \mathrm{s}$ and a mean free path of $L_{m}=$ $6 \mu \mathrm{m}$ at a saturation hole density of $p=6 \times 10^{11} \mathrm{~cm}^{-2}$ [25], suggesting that our junctions are in the ballistic limit. The superconducting coherence length in the ballistic limit is given by $\xi_{\mathrm{N}}=\left(\hbar v_{F}\right) / \Delta_{0}$, with $v_{F}$ being the Fermi velocity in the semiconductor and $\Delta_{0}=0.2 \mathrm{meV}$ being the gap size in the superconducting lead. Using the effective mass $m^{*}=0.09 m_{e}$ [25], we estimate the Fermi velocity to be $v_{F} \approx 2.3 \times 10^{7}$ $\mathrm{cm} / \mathrm{s}$, giving $\xi_{\mathrm{N}} \approx 770 \mathrm{~nm}$. We note that the switching current of a ballistic superconductor-semiconductor-superconductor (SNS) junction is expected to saturate for junction lengths shorter than the superconducting coherence length $\xi_{\mathrm{N}}$ at low temperature [34]. The fact that we do not observe this suggests either an unusually small $\xi_{\mathrm{N}}$ or a larger effective junction length, as will be discussed below.

\section{B. DC and AC Josephson effect}

To verify the Josephson nature of the supercurrent, we perform phase-sensitive experiments. Figure 2(a) shows a color plot of the source-drain resistance $(d V / d I)$ as a function of the external out-of-plane magnetic field $B$ for a junction with $L=450 \mathrm{~nm}$ and $W=1.5 \mu \mathrm{m}$. The clear modulation of the switching current, corresponding to the edge of the black area in the color plot, follows a clear Fraunhofer-like pattern, demonstrating the dc Josephson effect. It should be noted that the supercurrent does not drop to zero at integer flux quanta, which we attribute to an asymmetric current distribution in the device [35]. In addition, we observe multiple steps above $I_{\mathrm{S}}$ that are linearly spaced in voltage. The first step can be seen as the resistance peak at higher bias in Fig. 2(a). We speculate that these steps originate from finite coupling of the junction to some cavity mode, with a frequency of $f \approx 100 \mathrm{MHz}$, in or outside the device.

Based on the junction area and taking into account the increased thin-film penetration depth $\lambda_{\text {film }} \approx 105 \mathrm{~nm}$, as discussed in the Supplemental Material (SM) [36], one would expect a single flux quantum $\Phi_{0}$ through the junction at $B=2 \mathrm{mT}$, a magnetic field $\sim 3$ times larger than measured in Fig. 2. This deviation suggests significant flux focusing due to the superconducting aluminum leads next to the junction. We further observe that the Fraunhofer period increases with increasing magnetic field, and this could be explained from 
(a)
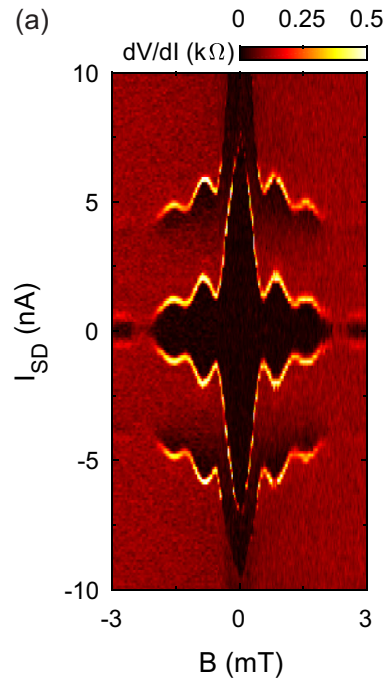

(b)

$$
10
$$

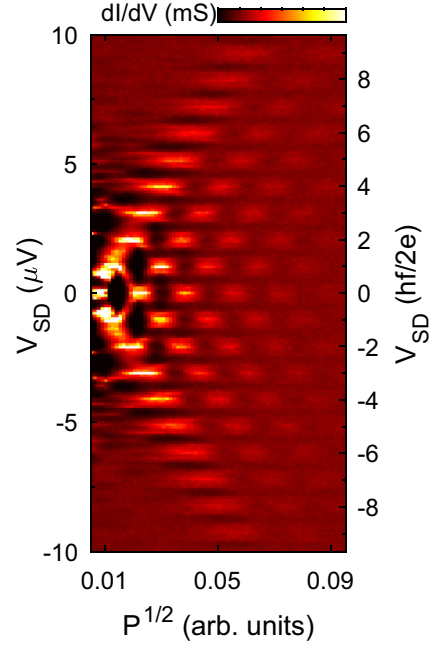

FIG. 2. (a) Color map of the differential resistance $d V / d I$ of the junction as a function of magnetic field $B$ and bias voltage $V_{\mathrm{SD}}$ at $V_{\mathrm{TG}}=-5 \mathrm{~V}$. A Fraunhofer-like modulation of the switching current as a function of $B$ is observed, confirming the Josephson nature of the devices. (b) Differential conductance $d I / d V$ of the junction as a function of bias current $I_{\mathrm{SD}}$ and microwave excitation amplitude $P^{1 / 2}$, showing clear Shapiro steps at $V_{\mathrm{SD}}=n h f / 2 e \approx n \times 1.03 \mu \mathrm{V}$, with excitation frequency $f=500 \mathrm{MHz}$.

reduced flux focusing as the Al layer is leaving the Meissner state.

In the presence of microwave irradiation, the ac Josephson effect gives rise to Shapiro steps in the source-drain voltage as a function of bias current. When applying an ac excitation with frequency $f=500 \mathrm{MHz}$ using an antenna near the junction with $L=50 \mathrm{~nm}$ and $W=1 \mu \mathrm{m}$, we observe clear peaks in the conductance [see Fig. 2(b)] at $V_{n}=n h f / 2 e=$ $n \times 1.03 \mu \mathrm{V}$, corresponding to plateaus in the source-drain voltage. We also observe small conductance peaks positioned at $\delta=0.22 \mu \mathrm{V}$ on either side of a Shapiro conductance peak. The steps are independent of applied microwave frequency and are observed in multiple junctions. These steps are not yet understood but may have the same origin as the steps observed without microwave radiation.

\section{Multiple Andreev reflections}

In an SNS system, multiple Andreev reflections (MARs) are expected to lead to resonances in the conductance at finite, subgap bias voltages $V_{m}=2 \Delta / m e$, with $m$ being the number of Andreev reflections and $\Delta$ being the superconducting gap of the superconductor. We observe kinks in the differential resistance that present as resistance peaks at elevated temperatures [see Fig. 3(a)], which we attribute to MARs and investigate by plotting the numerical derivative of the device resistance to extract the peak positions as a function of temperature [see Figs. 3(b) and 3(c)]. For a tunnel contact, one would expect MARs to cause a dip in the resistance, while for transparent contacts higher-order Andreev reflections still contribute significantly and MARs can cause a peak in resistance [37], as recently measured for an epitaxial aluminum/indium arsenide junction [38]. By
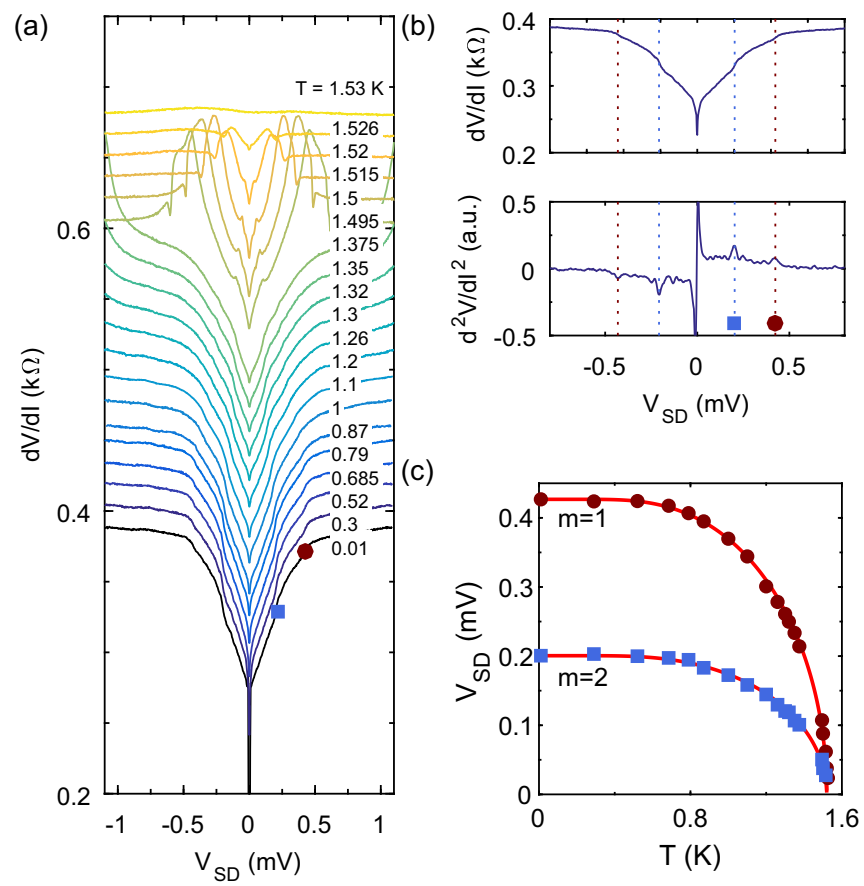

FIG. 3. (a) Temperature dependence of the junction resistance at $V_{\mathrm{TG}}=-5.0 \mathrm{~V}$. The traces are offset for clarity. (b) Resistance curve taken at $0.3 \mathrm{~K}$ (top panel) and its numerical derivative (bottom panel). The dotted lines show the position of the first and second MARs. Below $1.5 \mathrm{~K}$ their position is determined by the peaks in the numerical derivative, while above $1.5 \mathrm{~K}$ clear resistance peaks are visible. (c) Temperature dependence of the first and second MAR features. Red lines are BCS fits scaled to match the MAR position at $T \approx 10 \mathrm{mK}$.

analyzing the excess current, as seen from the conductance enhancement in Fig. 3(a) and details in the SM, we find a junction transparency $T$ between 0.6 and 0.7 , consistent with the small peak observed in the resistance due to MARs [38]. The MAR feature disappears when the temperature is raised above the aluminum film critical temperature $T_{\mathrm{C}}=1.52 \mathrm{~K}$. We fit the data in Fig. 3(c) with a BCS-like gap as obtained from a self-consistent solution of the gap equation, scaled to the MAR positions at $10 \mathrm{mK}$ [38] and using the Al film critical temperature. We find $\Delta=0.2 \mathrm{meV}$, as extracted from the low-temperature value of the $m=1$ MAR feature. The data suggest that $V_{m=2} \neq 2 V_{m=1}$, which may be a result of the resistance not peaking at exactly $V=2 \Delta / m e$ [38] or our analysis of the derivative of the resistance. This is also supported by the slight mismatch between the derived gap from the $m=1$ MAR feature and from the observed critical temperature $\Delta_{\mathrm{BCS}}=1.76 k_{B} T_{\mathrm{C}}=0.23 \mathrm{meV}$, with $k_{B}$ being Boltzmann's constant.

At very high bias voltages, we observe another resistance peak, as can be seen in the traces for $T>1.49 \mathrm{~K}$ in Fig. 3(a). At base temperature, the peak is observed at $V_{\mathrm{SD}}=2 \mathrm{mV}$, which is a bias voltage $\sim 10$ times above the observed superconducting gap (see SM, Fig. S2). The peak shifts to lower bias voltages with increasing temperature and disappears at $T_{\mathrm{C}}$, indicating it has a superconducting origin. A similar peak was observed before [38-40] and was attributed 
(a)

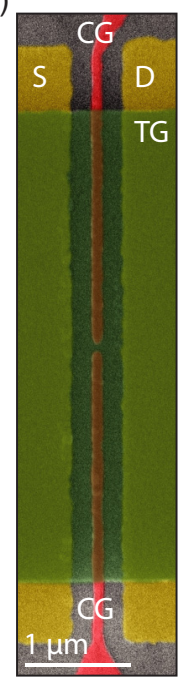

(b)

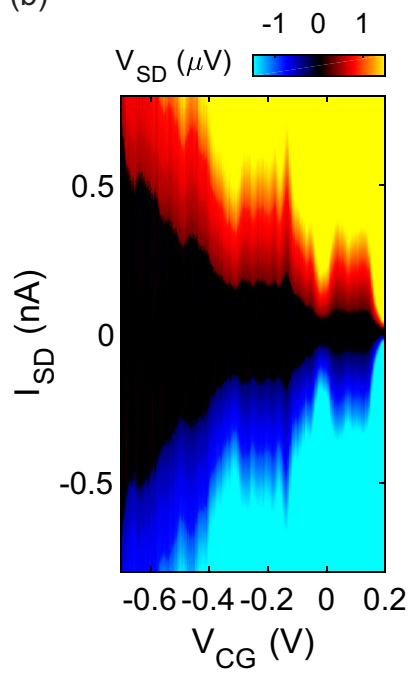

(c)

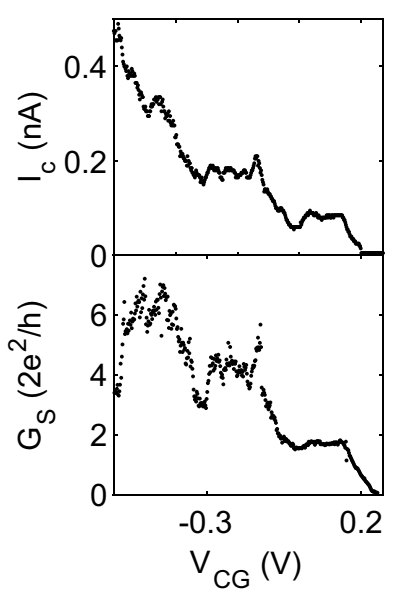

(d)

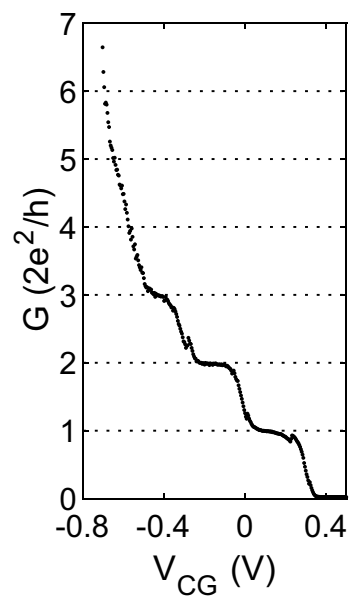

FIG. 4. (a) False-color SEM image of the superconducting QPC. A set of constriction gates (CG, illustrated in red) is added to confine the number of transport channels through the junction. (b) Color plot showing the effect of CG on the $I V$ curve of the device, measured at $V_{\mathrm{TG}}=-2.8 \mathrm{~V}$. Clear plateaus in the switching current are observed, demonstrating the supercurrent discretization due to the discrete number of modes in the SQPC. The additional oscillations are reproducible and likely an effect of Fabry-Pérot interference. (c) Discretization of switching current $I_{\mathrm{S}}$ (top panel) and subgap conductance $G_{\mathrm{S}}$ (bottom panel), demonstrating the ballistic nature of the superconducting device. Andreev reflection causes an enhanced conductance, resulting in steps exceeding $2 e^{2} / h$. (d) Normal-state QPC conductance $G_{\mathrm{N}}$ as a function of constriction gate voltage $V_{\mathrm{CG}}$ in an out-of-plane magnetic field sufficiently large to drive the aluminum Ohmic leads into the normal state. For $V_{\mathrm{C}}>-0.6 \mathrm{~V}$, the device conductance quantizes in steps of $G_{0}=2 e^{2} / h$. For $V_{\mathrm{C}}<-0.6 \mathrm{~V}$, a current can flow underneath the constriction gates, resulting in a large conductance.

to nonequilibrium effects appearing in planar junctions where the high-mobility 2DHG extends underneath the superconducting contacts. Such an extended interface may increase the probability of Andreev reflection and could thereby be a contribution to the observed transparent superconductorsemiconductor interfaces in planar structures.

\section{SUPERCONDUCTING QUANTUM POINT CONTACTS}

An important aspect for hybrid devices is whether transport can be restricted to individual channels. Quantum point contacts (QPCs) form an excellent playground to study the quantized nature of conductance. In germanium, this was recently used to measure the strong $g$-factor anisotropy of heavy holes in strained $\mathrm{SiGe} / \mathrm{Ge} / \mathrm{SiGe}$ heterostructures [41]. Here, we focus on superconducting QPCs (SQPCs), which are predicted to give rise to a discretization of the switching current in a superconducting junction [42,43]. Signatures of discrete supercurrents have been observed in InAs heterostructures [44-46] and Si/Ge nanowires [28].

To study this effect in planar germanium, we have fabricated devices similar to the junctions discussed above, but with a set of additional constriction gates (CGs), allowing us to deplete the 2DHG locally. The accumulation TG overlaps the CG but is electrically isolated by a 17-nm ALD-grown $\mathrm{Al}_{2} \mathrm{O}_{3}$ layer. Figures 4(b) and 4(c) show the transport characteristics of such a device. We tune the constriction gates to be more positive than the TG. Upon increasing the voltage $V_{\mathrm{CG}}$ we see that the conductance is reduced as, first, the transport underneath the constriction gates is turned off [see Fig. 4(d) for $V_{\mathrm{CG}}<-0.7 \mathrm{~V}$ ] and, for large enough positive voltages $\left(V_{\mathrm{CG}}>0.3 \mathrm{~V}\right)$, the current through the constriction also vanishes.

In the intermediate regime, superconducting transport is carried by discrete modes, yielding clear discretization of the supercurrent and conductance. In Fig. 4(c), the extracted switching current is shown together with the subgap conductance $G_{\mathrm{s}}$, measured at a bias current of $I_{\mathrm{SD}}=0.9 \mathrm{nA}$ $>I_{\mathrm{S}}$, such that a finite voltage $0<V_{\mathrm{SD}}<\Delta$ drops over the junction for all values of $V_{\mathrm{CG}}$. The supercurrent increases in steps $\delta I_{\mathrm{S}}=85 \mathrm{pA}$. On top of the supercurrent discretization, we also observe oscillations in the switching current and subgap conductance. We ascribe these to Fabry-Pérot-like interference effects in the QPC [47]. Finite scattering at the constriction interfaces most likely causes the oscillations and may also be related to the reduced QPC $I_{\mathrm{S}} R_{\mathrm{N}}=1.1 \mu \mathrm{V}$ that we observe for these devices, compared to the JoFET $I_{\mathrm{S}} R_{\mathrm{N}}=$ $17 \mu \mathrm{V}$ [see Fig. 1(c)]. In the short and low-temperature limit, each mode in the QPC is expected to contribute $\delta I_{\mathrm{S}}=e \Delta / \hbar=$ $49 \mathrm{nA}$, about three orders of magnitude larger than measured in our SQPC. This is consistent with the observed SQPC $I_{\mathrm{S}} R_{\mathrm{N}}$ also being approximately three orders of magnitude smaller than the expected $I_{\mathrm{S}} R_{\mathrm{N}}=\pi \Delta / e=0.63 \mathrm{meV}$ for a short and ballistic junction in the low-temperature limit [48], using the aluminum film superconducting gap $\Delta$ as obtained from the measured MAR.

Owing to the conductance doubling caused by the Andreev reflection, the subgap conductance can increase in steps with amplitude larger than $2 e^{2} / h$ [49], and we measure an average step height of $G_{\mathrm{S}}=3.4 e^{2} / h$. This can be compared to the normal-state conductance of the device $G_{\mathrm{N}}$ around zero bias current, measured at an out-of-plane field of 
several milliteslas, as shown in Fig. 4(d), where quantization in steps of $G_{\mathrm{N}}=2 e^{2} / h$ can be observed. Furthermore, we observe a dip structure in the conductance at the transition between the plateaus, which is not present in the normal-state conductance. This was also observed in InAs nanowires [50] and quantum wells [51] and is attributed to a strong subband mixing near the opening of a new channel due to the Van Hove singularity, canceling the Andreev enhancement of the conductance.

\section{DISCUSSION}

The observation of a relatively low $I_{\mathrm{S}} R_{\mathrm{N}}$ product provides room for further investigation and possible optimization. A possible reason could be the presence of a strong suppression of the induced superconducting gap as a result of the diffusion process of the $\mathrm{Al}$ contacts. Research could thus focus on epitaxial interfaces in order to reduce contact transparency, although we already find rather high transparency from excess current measurements. Alternatively, it may also be that transport is in the long-junction limit, even for the shortest junctions, with a corresponding reduced $I_{\mathrm{S}} R_{\mathrm{N}}$ [48]. This would also explain the pure exponential length dependence of the switching current we measure for junction lengths down to $50 \mathrm{~nm}$, typically observed in long junctions. A possible origin could be an extended interface that propagates underneath the Al contacts. This increased effective junction length could also explain the observed resistance peak at high bias. Alternatively, the coherence length could be unexpectedly short, for example, due to transport carried by heavy holes with spin $J=3 / 2$, strong spin-orbit coupling, and very anisotropic $g$-factors.

While these speculations provide avenues for future research, the experimentally measured $I_{\mathrm{S}} R_{\mathrm{N}}=17 \mu \mathrm{V}$ already exceeds significantly the thermal energy at base temperature and clearly demonstrates proximity superconductivity in planar germanium. The gate-tunable Josephson supercurrent ranging over micrometer length scales provides great opportunities for hybrid superconductor-semiconductor devices. Single-channel transport as demonstrated in the superconducting quantum point contact provides further scope for experiments requiring individual modes, such as Andreev spectroscopy of the superconducting gap. Planar gate-tunable superconducting qubits are within reach and could be coupled to nuclear spin-free spin qubits fabricated on the same platform. Topological qubits may require further development such as the demonstration of a hard gap but could profit from the large $g$-factor of heavy holes and from the low-disorder environment found in our systems. These demonstrations are an essential building block for the development of hybrid technologies and show that germanium is a strong candidate for novel quantum electronic devices.

\section{ACKNOWLEDGMENT}

We thank W. Lawrie for valuable discussions. M.V. acknowledges support through a FOM Projectruimte of the Foundation for Fundamental Research on Matter (FOM), associated with the Netherlands Organisation for Scientific Research (NWO).
[1] Y. Nakamura, Y. A. Pashkin, and J. S. Tsai, Nature (London) 398, 786 (1999).

[2] J. R. Petta, A. C. Johnson, J. M. Taylor, E. A. Laird, A. Yacoby, M. D. Lukin, C. M. Marcus, M. P. Hanson, and A. C. Gossard, Science 309, 2180 (2005).

[3] L. DiCarlo, J. M. Chow, J. M. Gambetta, L. S. Bishop, B. R. Johnson, D. I. Schuster, J. Majer, A. Blais, L. Frunzio, S. M. Girvin, and R. J. Schoelkopf, Nature (London) 460, 240 (2009).

[4] T. F. Watson, S. G. J. Philips, E. Kawakami, D. R. Ward, P. Scarlino, M. Veldhorst, D. E. Savage, M. G. Lagally, M. Friesen, S. N. Coppersmith, M. A. Eriksson, and L. M. K. Vandersypen, Nature (London) 555, 633 (2018).

[5] C. Song, K. Xu, W. Liu, C. P. Yang, S. B. Zheng, H. Deng, Q. Xie, K. Huang, Q. Guo, L. Zhang, P. Zhang, D. Xu, D. Zheng, X. Zhu, H. Wang, Y. A. Chen, C. Y. Lu, S. Han, and J. W. Pan, Phys. Rev. Lett. 119, 180511 (2017).

[6] K. Itoh, W. L. Hansen, E. E. Haller, J. W. Farmer, V. I. Ozhogin, A. Rudnev, and A. Tikhomirov, J. Mater. Res. 8, 1341 (1993).

[7] K. M. Itoh and H. Watanabe, MRS Commun. 4, 143 (2014).

[8] M. Veldhorst, J. C. C. Hwang, C. H. Yang, A. W. Leenstra, B. de Ronde, J. P. Dehollain, J. T. Muhonen, F. E. Hudson, K. M. Itoh, A. Morello, and A. S. Dzurak, Nat. Nanotechnol. 9, 981 (2014).

[9] A. J. Sigillito, R. M. Jock, A. M. Tyryshkin, J. W. Beeman, E. E. Haller, K. M. Itoh, and S. A. Lyon, Phys. Rev. Lett. 115, 247601 (2015).
[10] T. W. Larsen, K. D. Petersson, F. Kuemmeth, T. S. Jespersen, P. Krogstrup, J. Nygård, and C. M. Marcus, Phys. Rev. Lett. 115, 127001 (2015).

[11] G. de Lange, B. van Heck, A. Bruno, D. J. van Woerkom, A. Geresdi, S. R. Plissard, E. P. A. M. Bakkers, A. R. Akhmerov, and L. DiCarlo, Phys. Rev. Lett. 115, 127002 (2015).

[12] X. Mi, M. Benito, S. Putz, D. M. Zajac, J. M. Taylor, G. Burkard, and J. R. Petta, Nature (London) 555, 599 (2018).

[13] N. Samkharadze, G. Zheng, N. Kalhor, D. Brousse, A. Sammak, U. C. Mendes, A. Blais, G. Scappucci, and L. M. K. Vandersypen, Science 359, 1123 (2018).

[14] A. J. Landig, J. V. Koski, P. Scarlino, U. C. Mendes, A. Blais, C. Reichl, W. Wegscheider, A. Wallraff, K. Ensslin, and T. Ihn, Nature (London) 560, 179 (2018).

[15] J. D. Sau, R. M. Lutchyn, S. Tewari, and S. Das Sarma, Phys. Rev. Lett. 104, 040502 (2010).

[16] J. Alicea, Phys. Rev. B 81, 125318 (2010).

[17] S. Hoffman, C. Schrade, J. Klinovaja, and D. Loss, Phys. Rev. B 94, 045316 (2016).

[18] M. Leijnse and K. Flensberg, Phys. Rev. Lett. 107, 210502 (2011).

[19] M. Leijnse and K. Flensberg, Phys. Rev. B 86, 104511 (2012).

[20] C. Morrison, J. Foronda, P. Wiśniewski, S. D. Rhead, D. R. Leadley, and M. Myronov, Thin Solid Films 602, 84 (2016). 
[21] M. Failla, J. Keller, G. Scalari, C. Maissen, J. Faist, C. Reichl, W. Wegscheider, O. J. Newell, D. R. Leadley, M Myronov, and J. Lloyd-Hughes, New J. Phys. 18, 113036 (2016).

[22] N. W. Hendrickx, D. P. Franke, A. Sammak, M. Kouwenhoven, D. Sabbagh, L. Yeoh, R. Li, M. L. V. Tagliaferri, M. Virgilio, G. Capellini, G. Scappucci, and M. Veldhorst, Nat. Commun. 9, 2835 (2018).

[23] H. Watzinger, J. Kukučka, L. Vukušić, F. Gao, T. Wang, F. Schäffler, J.-J. Zhang, and G. Katsaros, Nat. Commun. 9, 3902 (2018).

[24] L. Vukušić, J. Kukučka, H. Watzinger, J. M. Milem, F. Schäffler, and G. Katsaros, Nano Lett. 18, 7141 (2018).

[25] A. Sammak, D. Sabbagh, N. W. Hendrickx, M. Lodari, B. P. Wuetz, A. Tosato, L. Yeoh, M. Bollani, M. Virgilio, M. A. Schubert, P. Zaumseil, G. Capellini, M. Veldhorst, and G. Scappucci, Adv. Funct. Mater. 0, 1807613 (2019).

[26] L. A. Terrazos, E. Marcellina, S. N. Coppersmith, M. Friesen, A. R. Hamilton, X. Hu, B. Koiller, A. L. Saraiva, D. Culcer, and R. B. Capaz, arXiv:1803.10320.

[27] G. Katsaros, P. Spathis, M. Stoffel, F. Fournel, M. Mongillo, V. Bouchiat, F. Lefloch, A. Rastelli, O. G. Schmidt, and S. D. Franceschi, Nat. Nanotechnol. 5, 458 (2010).

[28] J. Xiang, A. Vidan, M. Tinkham, R. M. Westervelt, and C. M. Lieber, Nat. Nanotechnol. 1, 208 (2006).

[29] F. Vigneau, R. Mizokuchi, D. C. Zanuz, X. Huang, S. Tan, R. Maurand, S. Frolov, A. Sammak, G. Scappucci, F. Lefloch, and S. De Franceschi, Nano Lett. 19, 1023 (2019).

[30] V. E. Calado, S. Goswami, G. Nanda, M. Diez, A. R. Akhmerov, K. Watanabe, T. Taniguchi, T. M. Klapwijk, and L. M. K. Vandersypen, Nat. Nanotechnol. 10, 761 (2015).

[31] Z. Wan, A. Kazakov, M. J. Manfra, L. N. Pfeiffer, K. W. West, and L. P. Rokhinson, Nat. Commun. 6, 7426 (2015).

[32] W. Yu, Y. Jiang, C. Huan, X. Chen, Z. Jiang, S. D. Hawkins, J. F. Klem, and W. Pan, Appl. Phys. Lett. 105, 192107 (2014).

[33] F. Qu, F. Yang, J. Shen, Y. Ding, J. Chen, Z. Ji, G. Liu, J. Fan, X. Jing, C. Yang, and L. Lu, Sci. Rep. 2, 339 (2012).

[34] I. O. Kulik and A. N. Omel'yanchuk, Fiz. Nizk. Temp. 3, 945 (1977) [Sov. J. Low. Temp. Phys. 37, 459 (1977)].

[35] R. C. Dynes and T. A. Fulton, Phys. Rev. B 3, 3015 (1971).

[36] See Supplemental Material at http://link.aps.org/supplemental/ 10.1103/PhysRevB.99.075435 for an analysis of characteristic lengths in Al, excess current analysis, and additional high-bias spectroscopy data, which include Refs. [52-57].

[37] D. Averin and A. Bardas, Phys. Rev. Lett. 75, 1831 (1995).
[38] M. Kjaergaard, H. J. Suominen, M. P. Nowak, A. R. Akhmerov, J. Shabani, C. J. Palmstrøm, F. Nichele, and C. M. Marcus, Phys. Rev. Appl. 7, 034029 (2017).

[39] C. Nguyen, H. Kroemer, and E. L. Hu, Phys. Rev. Lett. 69, 2847 (1992).

[40] C. Nguyen, H. Kroemer, and E. L. Hu, Appl. Phys. Lett. 65, 103 (1994).

[41] R. Mizokuchi, R. Maurand, F. Vigneau, M. Myronov, and S. De Franceschi, Nano Lett. 18, 4861 (2018).

[42] C. W. J. Beenakker and H. van Houten, Phys. Rev. Lett. 66, 3056 (1991).

[43] A. Furusaki, H. Takayanagi, and M. Tsukada, Phys. Rev. Lett. 67, 132 (1991).

[44] H. Takayanagi, T. Akazaki, and J. Nitta, Phys. Rev. Lett. 75, 3533 (1995).

[45] T. Bauch, E. Hurfeld, V. M. Krasnov, P. Delsing, H. Takayanagi, and T. Akazaki, Phys. Rev. B 71, 174502 (2005).

[46] H. Irie, Y. Harada, H. Sugiyama, and T. Akazaki, Phys. Rev. B 89, 165415 (2014).

[47] A. Furusaki, H. Takayanagi, and M. Tsukada, Phys. Rev. B 45, 10563 (1992).

[48] M. Tinkham, Introduction to Superconductivity (McGraw Hill, New York, 2004).

[49] C. W. J. Beenakker, Rev. Mod. Phys. 69, 731 (1997).

[50] H. Zhang, O. Gül, S. Conesa-Boj, M. P. Nowak, M. Wimmer, K. Zuo, V. Mourik, F. K. de Vries, J. van Veen, M. W. A. de Moor, J. D. S. Bommer, D. J. van Woerkom, D. Car, S. R. Plissard, E. P. A. M. Bakkers, M. Quintero-Pérez, M. C. Cassidy, S. Koelling, S. Goswami, K. Watanabe, T. Taniguchi, and L. P. Kouwenhoven, Nat. Commun. 8, 16025 (2017).

[51] M. Kjaergaard, F. Nichele, H. J. Suominen, M. P. Nowak, M. Wimmer, A. R. Akhmerov, J. A. Folk, K. Flensberg, J. Shabani, C. J. Palmstrøm, and C. M. Marcus, Nat. Commun. 7, 12841 (2016).

[52] J. J. Hauser, J. Low Temp. Phys. 7, 335 (1972).

[53] H. J. Suominen, J. Danon, M. Kjaergaard, K. Flensberg, J. Shabani, C. J. Palmstrøm, F. Nichele, and C. M. Marcus, Phys. Rev. B 95, 035307 (2017).

[54] N. Klein, H. Chaloupka, G. Müller, S. Orbach, H. Piel, B. Roas, L. Schultz, U. Klein, and M. Peiniger, J. Appl. Phys. 67, 6940 (1990).

[55] A. I. Gubin, K. S. Il'in, S. A. Vitusevich, M. Siegel, and N. Klein, Phys. Rev. B 72, 064503 (2005).

[56] M. Octavio, M. Tinkham, G. E. Blonder, and T. M. Klapwijk, Phys. Rev. B 27, 6739 (1983).

[57] K. Flensberg, J. B. Hansen, and M. Octavio, Phys. Rev. B 38, 8707 (1988) 